\title{
Cardiovascular biomarkers predict susceptibility to lung injury in World Trade Center dust-exposed firefighters
}

\author{
Michael D. Weiden*\#,ף,\$, Bushra Naveed*,\$, Sophia Kwon*, Soo Jung Cho*, \\ Ashley L. Comfort", David J. Prezant ${ }^{\tau,+}$, William N. Rom ${ }^{\star}, \#$ and Anna Nolan*,\#,
}

ABSTRACT: Pulmonary vascular loss is an early feature of chronic obstructive pulmonary disease. Biomarkers of inflammation and of metabolic syndrome predict loss of lung function in World Trade Center (WTC) lung injury (LI). We investigated if other cardiovascular disease (CVD) biomarkers also predicted WTC-LI.

This nested case-cohort study used 801 never-smoker, WTC-exposed firefighters with normal pre-9/11 lung function presenting for subspecialty pulmonary evaluation (SPE) before March 2008. A representative subcohort of 124 out of 801 subjects with serum drawn within 6 months of 9/11 defined CVD biomarker distribution. Post-9/11 forced expiratory volume in $1 \mathrm{~s}(\mathrm{FEV} 1)$ at defined cases were as follows: susceptible WTC-LI cases with FEV $1 \leqslant 77 \%$ predicted (66 out of 801 ) and resistant WTC-LI cases with FEV $1 \geqslant 107 \%$ predicted (68 out of 801 ). All models were adjusted for WTC exposure intensity, body mass index at SPE, age on 9/11 and pre-9/11 FEV1.

Susceptible WTC-LI cases had higher levels of apolipoprotein-AII, C-reactive protein and macrophage inflammatory protein-4 with significant relative risks (RRs) of $3.85,3.93$ and 0.26 , respectively, with an area under the curve (AUC) of 0.858 . Resistant WTC-LI cases had significantly higher soluble vascular cell adhesion molecule and lower myeloperoxidase, with RRs of 2.24 and 2.89 , respectively (AUC 0.830 ).

Biomarkers of CVD in serum 6 months post-9/11 predicted either susceptibility or resistance to WTC-LI. These biomarkers may define pathways either producing or protecting subjects from pulmonary vascular disease and associated loss of lung function after an irritant exposure.

KEYWORDS: Airway inflammation, cytokines, pulmonary function testing

ome of the hallmarks of particulate matter (PM) exposure are systemic inflammation, endothelial dysfunction and subsequent end-organ damage [1-5]. Epidemiological investigation has documented associations between increased ambient PM, lung disease and cardiovascular disease (CVD) [6-12]. Inflammation and remodelling are key features of airflow obstruction in asthma and chronic obstructive pulmonary disease (COPD) [13, 14]. High ambient PM exposures significantly decrease forced expiratory volume in $1 \mathrm{~s}$ (FEV1) even after 5-7 days [15-17]. Systemic inflammation produces vascular endothelial injury and subsequent CVD [1820]. Recent studies associate systemic vascular involvement with lung disease [21, 22] and prospective studies have demonstrated an association between impaired lung function and central arterial stiffness even before the development of CVD, with systemic inflammation contributing to this association [23-25].

Recently, we have shown that biomarkers of inflammation (granulocyte-macrophage colonystimulating factor and macrophage-derived chemokine) and of the metabolic syndrome, observed in serum drawn within 6 months of World Trade Center (WTC) exposure, predict the post-9/11 decline in FEV1 in this cohort of WTC-exposed Fire Department of the City of New York (FDNY) firefighters $[3,17]$. Utilising a nested case-cohort design, the goal of this study was to investigate whether CVD serum biomarkers drawn within 6 months of 9/11 can predict WTC-lung injury (LI) in this longitudinal well phenotyped cohort of FDNY firefighters. We hypothesised that individuals exposed to WTC particulates who went on to

For editorial comments see page 999.

AFFILIATIONS

*Division of Pulmonary, Critical Care and Sleep, New York University, School of Medicine New York, New York, NY,

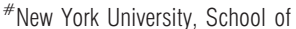
Medicine, Dept of Environmental Medicine, Tuxedo Park, NY "Bureau of Health Services and Office of Medical Affairs, Fire Dept of New York, New York, NY, and +Pulmonary Medicine Division, Dept of Medicine, Montefiore Medical Center and Albert Einstein College of Medicine, New York, NY, USA.

${ }^{\S}$ These authors contributed equally to this study.

CORRESPONDENCE

A. Nolan

Division of Pulmonary, Critical Care and Sleep

New York University School of Medicine

5501 st Avenue

NB 7N24

New York

NY 10016

USA

E-mail: anna.nolan@med.nyu.edu

Received:

May 142012

Accepted after revision:

Aug 052012

First published online:

Aug 162012 
develop persistent WTC-LI, would express different levels of CVD biomarkers than those similarly exposed individuals who were resistant to developing WTC-LI.

The collapse of the WTC exposed tens of thousands of people to extremely high PM concentrations. Several cohorts of WTCexposed individuals have been longitudinally followed. Among exposed workers, volunteers and lower Manhattan residents, abnormal spirometry has been a common finding [26]. In rescue/recovery workers from the FDNY, WTC exposure led to WTC-LI, as proven by substantial declines in pulmonary function in the first 6 months after $9 / 11$ (at a rate 12 times greater than that found prior to 9/11) [27, 28], and these findings persisted over the next 6.5 years [29].

Pulmonary vascular injury occurs early in smoking-related chronic obstructive lung disease (COPD) with pulmonary perfusion abnormalities and reduced blood return to the heart observed prior to development of abnormal FEV1 [30, 31]. Similar pathophysiology probably occurs in irritant-induced COPD. Pulmonary arteriopathy was present in $58 \%$ of lung biopsies from non-FDNY WTC-exposed individuals and in $74 \%$ with constrictive bronchiolitis after inhalational exposures during military service in Iraq and Afghanistan [32, 33].

\section{METHODS}

\section{Study participants}

WTC-exposed FDNY firefighters entered the FDNY Medical Monitoring and Treatment Program (MMTP) and had spirometry at medical monitoring entry (MME) [34, 35]. Briefly, FDNY spirometry was performed according to American Thoracic Society (ATS)/European Respiratory Society (ERS) guidelines using Portascreen Spirometry (S\&M Instruments, Doylestown, PA, USA) [36]. In order to provide three acceptable spirograms, seated workers wearing nose clips performed up to eight forced expiratory manoeuvres per testing session. Subspecialty pulmonary examination (SPE) pulmonary function tests (PFTs) were performed according to ATS/ERS guidelines using a Jaeger Masterscreen PFT System (Viasys Healthcare, Yorba Linda, CA, USA). All acceptable measures were expressed in absolute values (litres) and as percentage predicted (\% pred) of normal.

The study cohort was derived from symptomatic subjects referred for SPE $(\mathrm{n}=1720)$ between October 1, 2001 and March 10, 2008 [37]. The baseline cohort consisted of never-smokers (consistently reported to be not smoking on all health screens), male, had reliable National Health and Nutrition Examination Survey (NHANES) normative data for predicted FEV1, had post-9/11 FDNY PFTs within 200 days of $9 / 11$, and had pre-9/11 FEV1 $>75 \%$ pred (801 (47\%) out of 1720$)$. All subjects signed informed institutional review board-approved consent at the time of enrolment allowing analysis of their information and samples for research (Montefiore Medical Center, New York, NY, USA, \#07-09-320 and New York University, New York, NY, USA, \#11-00439).

Cases were defined by their FEV1 \% pred at SPE using NHANES III criteria. Those resistant to WTC-LI were defined as being within one standard deviation of the highest FEV1 \% pred of the study cohort $(n=100)$ had an FEV1 $\geqslant 107 \%$ pred. Those susceptible to WTC-LI were similarly defined as being within one standard deviation of the lowest FEV $1 \%$ pred of the cohort $(n=100)$, which was an FEV1 $\leqslant 77 \%$ pred. The subcohort controls $(n=171)$ were randomly selected from the study cohort after stratification based on body mass index (BMI) and FEV1 \% pred at MME. Serum biomarkers were available for 124 out of 171 of the subcohort controls, 68 out of 100 resistant cases and 66 out of 100 susceptible cases.

\section{Demographics}

Age, sex and years of service at FDNY were obtained from the FDNY WTC monitoring database. BMIs were calculated from height and weight measured at the time of MME and SPE. Degree of exposure was self-reported at the first FDNY WTC monitoring and was categorised using the FDNY-WTC Exposure Intensity Index (arrival time): 1) present on the morning of September 11, 2001; 2) arrived after noon on September 11, 2001; and 3) arrived on September 12, 2001. Those arriving after day 3 were excluded from analysis as a result of their low numbers in this sample $[3,17]$.

\section{Serum sampling and analysis}

Blood was drawn at MME, from October 29, 2001 to January 31,2002 , for the subjects in this analysis. Samples were allowed to stand for $1 \mathrm{~h}$ at room temperature before being centrifuged at $1800 \times \mathrm{g}$ for $10 \mathrm{~min}$. Serum was stored at $-80^{\circ} \mathrm{C}$ (BioReference Laboratories, Inc., Elmwood Park, NJ, USA). Serum was thawed once at $4^{\circ} \mathrm{C}$ and assayed using cardiovascular disease (CVD)-1 (human cardiovascular disease (HCVD) 1-67AK), apolipoproteins (APO-62K), and neurodegenerative (human neurodegenerative (HND)G2-36K) panels according to manufacturer's instructions (Millipore, Billerica, MA, USA) on a Luminex 200IS (Luminex Corp., Austin, TX, USA). Data analysed with MasterPlex QT software (version 1.2; MiraiBio, Inc., Alameda, CA, USA). Each batch of samples processed contained controls and cases in an $\sim 12: 7$ ratio.

\section{Statistical analysis}

SPSS 19 (IBM, Armonk, NY, USA) used for all database management and statistics. Demographic information and analyte levels were compared by Mann-Whitney U-test or Kruskal-Wallis test where appropriate. We used logistic regression analysis to estimate the CVD biomarkers relative risks (RRs) for crude and single-analyte models adjusted for the confounders of age at 9/11, BMI at SPE, exposure group and pre-9/11 FEV1 \% pred (table 1). Relative risk was calculated by binary logistic regression. Cases (WTC-LI susceptible or resistant) were compared to the subcohort controls as the dichotomous outcome variable and cut-off points of analytes were used as the predictors. Myeloperoxidase (MPO) below the 25th percentile, macrophage inflammatory protein (MIP)- 4 on or above the 50th percentile, C-reactive protein (CRP) $\geqslant 2.45 \mathrm{mg} \cdot \mathrm{dL}^{-1}$ and apolipoprotein (Apo)-AII, soluble vascular cell adhesion molecule (sVCAM) on or above the 75th percentile were defined as cut-off points $[3,38,39]$. Models were adjusted for potential confounders; $\mathrm{BMI}$ at SPE, exposure group as categorical variable, age on September 11, 2001, and FEV1 \% pred prior to $9 / 11$ as a continuous variable. The Hosmer-Lemeshow goodness-of-fit statistic was used as a statistical test of model adequacy. Receiver operator characteristic (ROC) area under the curve (AUC) was also quantified for each final model. For all statistical tests, significance was assessed by a p-value $<0.05$. 


\section{TABLE 1 Values of significant serum analytes in single logistic regression model}

\begin{tabular}{|c|c|c|c|}
\hline Analyte & Resistant & Controls & p-value ${ }^{\#}$ \\
\hline Subjects $n$ & 68 & 124 & \\
\hline MPO $\mathrm{ng} \cdot \mathrm{mL}^{-1}$ & $117.94(68.01-176.91)$ & $143.56(105.26-212.31)$ & 0.016 \\
\hline $\mathrm{sVCAM} \mathbf{n g} \cdot \mathrm{mL}^{-1}$ & $1500.83(1126.57-1808.16)$ & $1349.97(1126.57-1568.65)$ & 0.103 \\
\hline
\end{tabular}

\section{RESULTS}

\section{Demographics}

We developed a subcohort control to represent the full spectrum of lung function response of the baseline cohort and two case definitions represented extremes of response to WTC dust and smoke (fig. 1). The demographics of all groups with serum available are summarised in table 2 . The control and case groups had similar WTC exposure, time from 9/11 to MME, time from $9 / 11$ to SPE, years of service and age at 9/11. BMI of susceptible cases was higher than controls or resistant cases at MME and SPE.

\section{Lung function}

Susceptible cases had lower FEV1 and this correlated with diffusing capacity of the lung for carbon monoxide (DLCO) and measures of airflow obstruction, including methacholine and bronchodilator response (table 3). Lung function in resistant cases and subcohort controls increased from the MME to SPE (105\% to $113 \%$ and $92 \%$ to $95 \%$, respectively) while the FEV1\% pred of affected cases continued to decline between the two pulmonary function tests $(79 \%$ pred to $72 \%$ pred; $p<0.001$ all comparisons).

\section{CVD biomarkers}

We measured CVD serum biomarkers expressed within 6 months of 9/11. Compared with subcohort controls, cases susceptible to WTC-LI had significant elevations of ApoAI (4273.98 versus $2569.94 \mu \mathrm{g} \cdot \mathrm{mL}^{-1}, \mathrm{p}=0.003$ ), ApoAII (1830.96 versus $\left.845.12 \mu \mathrm{g} \cdot \mathrm{mL}^{-1}, \mathrm{p}=0.002\right)$, ApoCII (305.80 versus $203.18 \mu \mathrm{g} \cdot \mathrm{mL}^{-1}$, $\mathrm{p}=0.006)$, ApoCIII (973.58 versus $\left.600.91 \mu \mathrm{g} \cdot \mathrm{mL}^{-1}, \mathrm{p}=0.005\right)$, and ApoE (257.43 versus $\left.140.71 \mu \mathrm{g} \cdot \mathrm{mL}^{-1}, \mathrm{p}=0.001\right)$, and significantly lower levels of MIP-4 (145.28 versus $\left.184.68 \mu \mathrm{g} \cdot \mathrm{mL}^{-1}, \mathrm{p}=0.015\right)$ (table 4). Whereas resistant WTC-LI cases had significantly decreased levels of MPO (117.94 versus $143.56 \mu \mathrm{g} \cdot \mathrm{mL}^{-1}$, $\mathrm{p}=0.016$ ) compared with subcohort controls (table 1).

\section{Resistant WTC-LI cases: development of RR models}

Univariate model

We found that MPO <25th percentile and sVCAM $\geqslant 75$ th percentile cases resistant to WTC-LI in both crude and confounder adjusted models.

\section{Multivariate model}

In a multivariate model that combined $\mathrm{sVCAM} \geqslant 1568.65 \mathrm{ng} \cdot \mathrm{mL}^{-1}$ and MPO $\leqslant 105.26 \mathrm{ng} \cdot \mathrm{mL}^{-1}$, both remained as significant predictors of resistance to WTC-LI RR 2.24 (95\% CI 1.07-4.70) and RR 2.89 (95\% CI 1.37-7.47), respectively (table 5). The final model for resistance to lung injury had an AUC of 0.830 (0.774-0.887).

\section{Susceptible WTC-LI cases: development of RR models}

Univariate analysis

We found that CRP $\geqslant 2.45 \mathrm{mg} \cdot \mathrm{dL}^{-1}$, ApoAI, ApoAII, ApoCII, ApoCIII and ApoE $\geqslant 75$ th $\%$ pred and MIP- $4 \geqslant 50 \%$ pred as crude values all significantly predicted the risk of having an FEV1 $\leqslant 77 \%$ pred at SPE in individuals who were exposed to WTC dust. CVD biomarkers predicted the risk of WTC-LI at SPE in both crude and confounder adjusted models (table 5).

\section{Multivariate model}

We then assessed the biomarkers' ability in the final logistic regression model to predict case status after adjusting for confounders. We found that a three-analyte model combining ApoAII, CRP and MIP-4 optimally predicted the susceptible cases with ApoAII $\geqslant 1772.87 \mu \mathrm{g} \cdot \mathrm{mL}^{-1}$ (RR 3.85, 95\% CI 1.798.26), $C R P \geqslant 2.45 \mathrm{mg} \cdot \mathrm{dL}^{-1}$ (RR 3.93, 95\% CI 1.43-10.79) and MIP-4 $>194.90 \mathrm{ng} \cdot \mathrm{mL}^{-1}$ (RR $0.26,95 \%$ CI $0.11-0.59$ ). The final model produced a ROC AUC of 0.858 (95\% CI 0.79-0.919) (table 5). ApoAII was used in the final model because it consistently produced the highest relative risk of any apolipoprotein when combined with another apolipoprotein and ApoAII made the other apolipoproteins insignificant when combined together.

\section{DISCUSSION}

We observed serum biomarkers classically associated with CVD to predict lung function after exposure to dust and smoke at the WTC site in FDNY nonsmoking firefighters with normal pre-9/11 lung function. Firefighters with elevated SVCAM and low MPO levels within 6 months of September 11, 2001, recovered lung function, returning to pre-9/11 values after an acute decline. Alternately, individuals with elevated ApoAII and CRP levels within 6 months of $9 / 11$ had significantly increased risk of developing decreased lung function over the subsequent 6 years, while elevated MIP-4 reduced the risk of susceptibility to decreased lung function.

Our observation that risk factors for vascular injury are also predictors of lung dysfunction is consistent with recent reports that perfusion abnormalities and reduced pulmonary blood flow occur prior to development of abnormal FEV1 in smokers at risk for COPD. In other WTC-exposed cohorts, pulmonary vasculopathy is a prominent feature. Vascular injury may be a prominent feature of irritant exposure, as soldiers exposed to sand and products of combustion also had vasculopathy on pathology. CRP is known a marker of acute systemic inflammation and CVD [40, 41]. It is biologically plausible that processes that injure systemic arteries could also injure pulmonary arteries. There is an inverse relationship between 


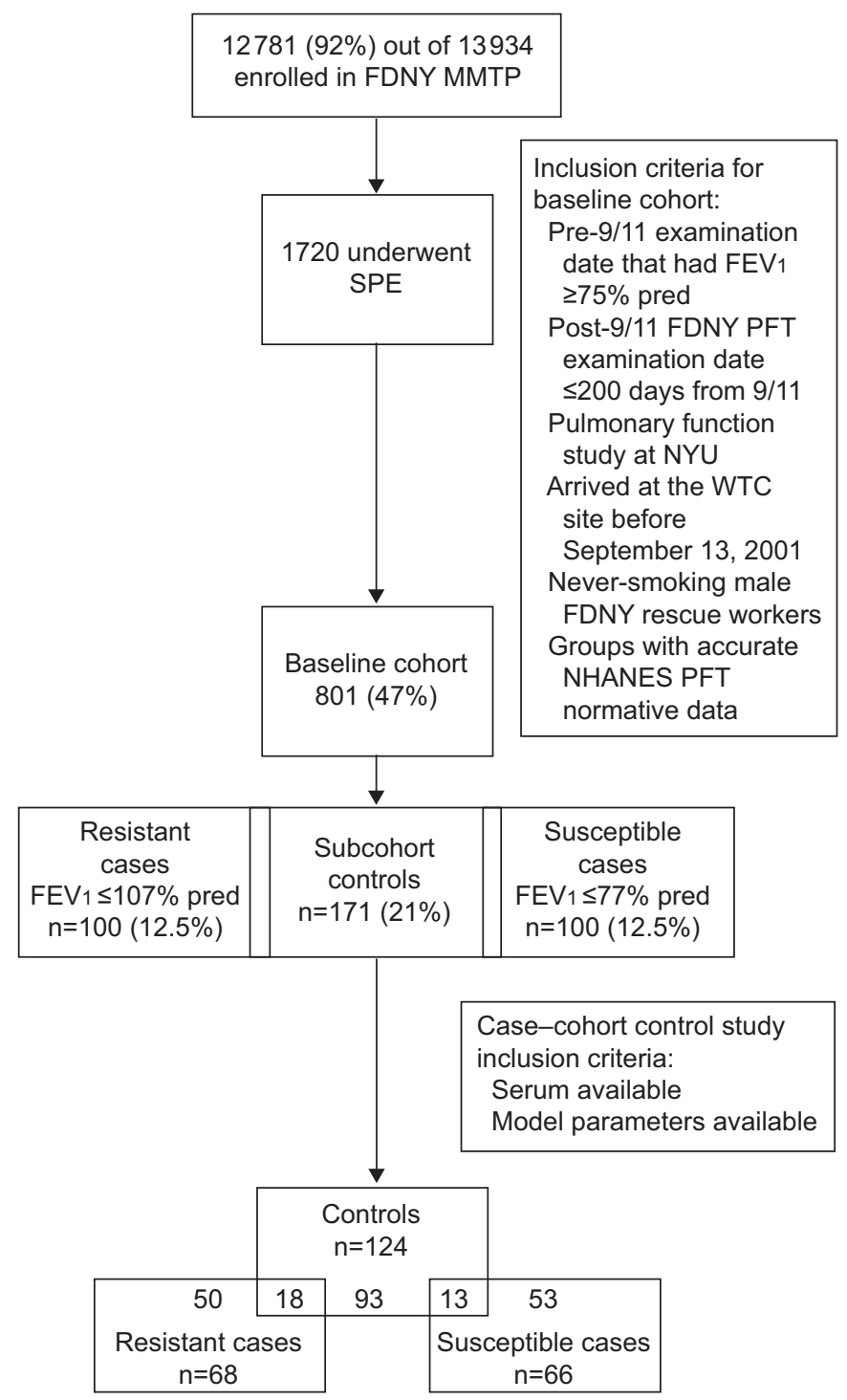

FIGURE 1. Study design. FDNY: Fire Department of the City of New York; MMTP: Medical Monitoring and Treatment Program; SPE: subspecialty pulmonary examination; 9/11: September 11, 2001; PFT: pulmonary function test; NYU: New York University; WTC: World Trade Center; NHANES: National Health and Nutrition Examination Survey; FEV1: forced expiratory volume in $1 \mathrm{~s}$; \% pred: \% predicted.

serum CRP and FEV1 [42]. CRP levels were elevated in individuals with COPD independent of any CVD risks [43]. Pulmonary hypertension, another disease of the pulmonary vasculature, is associated with CVD biomarkers [44]. This parallels our prior observation that dyslipidaemia predicts poor outcome after WTC dust exposure. MIP-4 (chemokine (C-C motif) ligand 18) is an early promoter of regulatory T-cell differentiation and may generate an anti-inflammatory counterregulatory response $[45,46]$. Our data demonstrate that low levels of MPO demonstrate less neutrophil activation in patients resistant to the damaging effects of WTC dust exposure. Neutrophil activation is an important mediator of the response to PM and induces pulmonary and cardiovascular vascular injury $[1,47]$. Taken together, the data in this and other recent reports emphasise the need to better understand the mechanisms by which inhaled irritants damage pulmonary vessels.
This case-cohort study design was utilised to assess the predictive abilities of CVD biomarkers in determining the relative risk of either being protected from or developing WTC-LI [48-50]. Subcohort controls, and susceptible and resistant cases were all drawn from the larger baseline cohort. The subcohort controls contain an overlapping population of those who also met case definitions. The odds ratios approximate the relative risk of the biomarker-disease relationship of the larger cohort. In addition, case-cohort studies have several advantages. First, comparing multiple case definitions to the same subcohort control group facilitates the identification of biomarkers of susceptibility and resistance to WTC-LI. Secondly, the case-cohort studies are cost-effective and logistically efficient, as biomarkers only needed to be assayed for a sample of the entire cohort. Lastly, the study design minimised batch bias and freeze-thaw problems associated with biomarker discovery [48].

The study cohort was nested within a larger group of intensively evaluated, longitudinally followed symptomatic firefighters who presented for SPE prior to March 2008. We chose to exclude ever-smokers to eliminate the confounding effect of smoking on lung function and CVD risk. We narrowed the baseline cohort of 801 to produce a subcohort control of 136 patients with serum available, a group large enough to be representative of the baseline cohort but small enough to allow cost effective measurement of biomarker expression. As the control group represented the larger cohort, comparing biomarker expression in cases with that in the subcohort control permitted relative risk measures produced by the biomarkers. We used FEV1 for case definition because it is the best single measure of lung function available on the entire FDNY cohort at each point of interest. Cases resistant to WTC-LI were defined by FEV1 in the top 12.5 percentile, while susceptible cases were defined by FEV1 in the lowest 12.5 percentile at SPE. This cohort had serial pulmonary function pre- and post-9/11, thereby allowing us to define resistance or susceptibility to WTC-LI after a severe irritant exposure. Another unique aspect of the study is biomarker expression was measured within 6 months of 9/11 and prior to recovery from injury or the development of persistent WTC-LI.

Cases susceptible or resistant to WTC-LI and the subcohort controls experienced similar exposure to smoke and dust, which led to a decline in their FEV1 within 6 months of 9/11. This suggests a similar response to acute irritant exposure. However, protected cases and cohort controls maintained normal lung function while affected cases developed abnormal FEV1 by SPE. One explanation for this may be that the WTC-LI cases had significantly lower pre-9/11 FEV1. Cases therefore needed a smaller relative decline to drop below $77 \%$ post- $9 / 11$. We adjusted for this potential confounder by adding pre- $9 / 11$ FEV1 to all models. Predictors of resistance and susceptibility were not strongly affected by pre-9/11 FEV1, demonstrating that the serum biomarker effect was independent of the preexposure lung function. Similarly, increased BMI is well known to be associated with reduced FEV1, but adjusting for this potential confounder did not alter the ability of the serum biomarkers to predict recovery or decline in lung function. This demonstrates an inflammatory process in susceptible lung injury cases that produces persistent loss of lung function for 
TABLE 2 Demographics and forced expiratory volume in $1 \mathrm{~s}(F E V 1)$ of subspecialty pulmonary evaluation cohorts

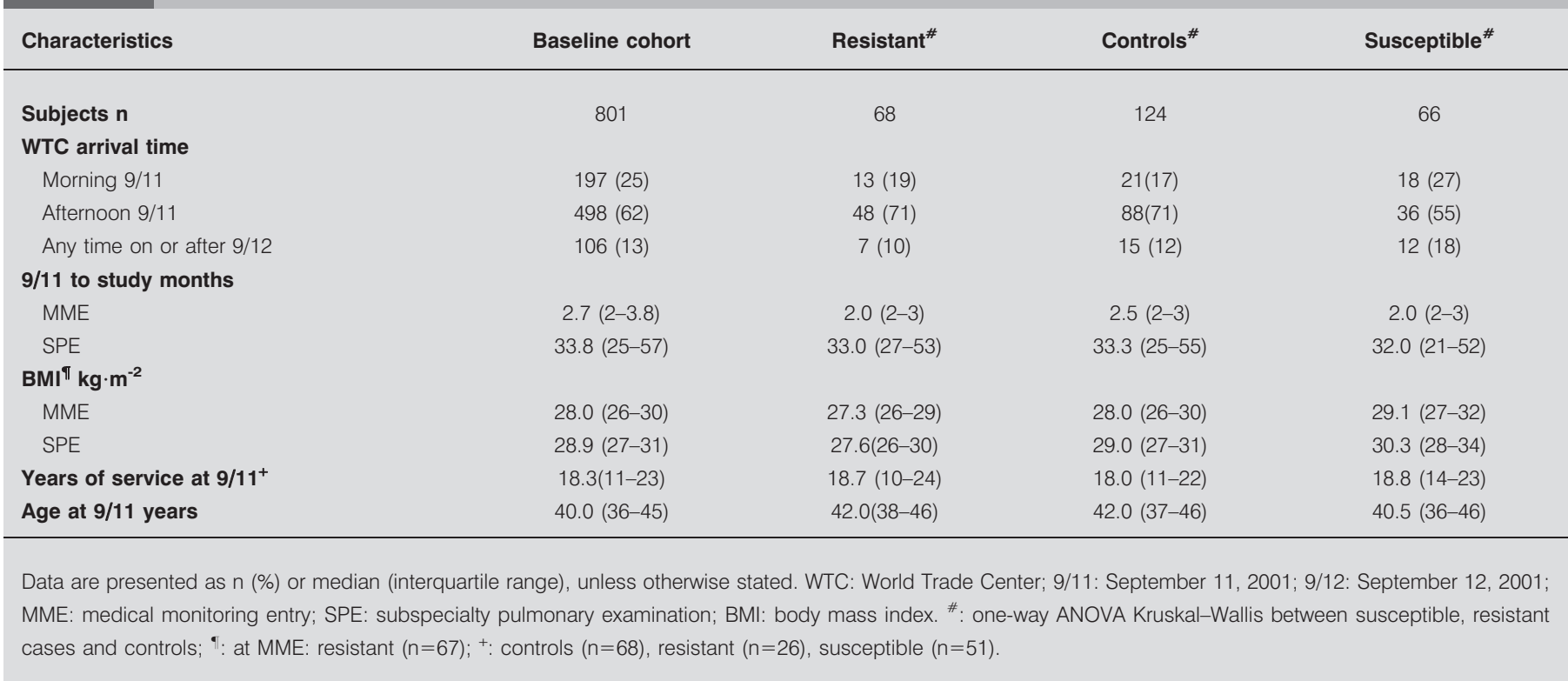

years after the insult. Conversely, resistant cases showed longterm protection from WTC-LI.

There are several limitations to this study. It comprises a single unique cohort of FDNY firefighters with serum samples available from October 29, 2001 until January 31, 2002. This limits the generalisability of these findings to other WTCexposed cohorts who probably include individuals with preexisting lung disease or other risk factors, such as smoking. Definitions of susceptible and resistant cases were based on FEV1 measured at SPE. We believe that low FEV1 is the single best outcome measure to define lung injury in the FDNY cohort because FEV1 has been longitudinally measured starting 3 years prior to September 11, 2001, and continues to be performed at every FDNY-WTC-MMTP. However, using FEV1 as a single measure of lung function could lead to nondifferential misclassification. As FEV1 is reduced in both restriction and obstruction, low FEV1 does not distinguish between the two. In a prior investigation, we have observed that obstruction caused the vast majority of abnormal FEV1 in WTCexposed fire fighters [37]. In addition, patients with accelerated decline in FEV1 who will progress to disease but currently have a normal FEV1 will be misclassified as controls. While misclassification may occur when using FEV $1<77 \%$ pred as a single measure of abnormal lung function, heterogeneity of disease(s) produced by this single measure will bias the results toward the null hypothesis. In spite of the potential for nondifferential information bias, using low FEV1 has yielded strong biomarkerdisease associations [3, 39]. As all the FDNY firefighters were exposed to WTC dust, we do not have unexposed control group to compare with and, therefore, we cannot determine if WTC exposure is necessary for the observed effect. Replication of these findings in other longitudinally followed populations with and without PM exposure will be important to demonstrate the generalisability of these findings.

TABLE 3 Pulmonary function testing

\begin{tabular}{|c|c|c|c|c|}
\hline & Resistant & Controls & Susceptible & p-value ${ }^{\#}$ \\
\hline Pre-9/11 FEV 1 & $117(107-124)$ & $102(92-112)$ & $89(82-96)$ & $<0.001$ \\
\hline \multicolumn{5}{|l|}{ SPE } \\
\hline $\mathrm{FEV}_{1}$ & $113(109-118)$ & $95(84-103)$ & $72(67-75)$ & $<0.001$ \\
\hline MCT slope & $0.039(0.02-0.07)$ & $0.048(0.03-0.12)$ & $0.15(0.05-1.8)$ & $<0.001$ \\
\hline
\end{tabular}

Data are presented as median (interquartile range), unless otherwise stated. 9/11: September 11, 2001; FEV1: forced expiratory volume in $1 \mathrm{~s}$; MME: medical monitoring entry; SPE: subspecialty pulmonary examination; MCT: methacholine challenge test; DLCO: diffusing capacity of the lung for carbon monoxide; \% pred \% predicted; BD: bronchodilator. ${ }^{\#}$ : significance was assessed by one-way ANOVA Kruskal-Wallis test between resistant and susceptible cases and controls: ": controls: $\mathrm{n}=94$; resistant cases: $n=57$; susceptible cases: $n=32{ }^{+}$: controls: $n=59$; resistant cases: $n=24$; susceptible cases: $n=44$. ${ }^{\text {s. }}$ controls: $n=64$; resistant cases: $n=26$; susceptible cases: $n=48$. 
TABLE 4 Values of significant serum analytes in single logistic regression model

\begin{tabular}{|c|c|c|c|}
\hline Analyte & Susceptible & Controls & p-value ${ }^{\#}$ \\
\hline Subjects $n$ & 66 & 124 & \\
\hline CRP $m g \cdot d L^{-1}$ & $6.28(3.28-13.16)$ & $5.10(1.99-11.19)$ & 0.151 \\
\hline MIP-4 $\mathrm{ng} \cdot \mathrm{mL}^{-1}$ & $145.28(106.56-211.25)$ & $184.68(109.68-948.20)$ & 0.008 \\
\hline ApoAl $\mu \mathrm{g} \cdot \mathrm{mL}^{-1}$ & 4273.98 (2039.29-6333.55) & 2569.94 (1833.34-4198.31) & 0.003 \\
\hline ApoAll $\mu \mathrm{g} \cdot \mathrm{mL}^{-1}$ & $1830.96(570.65-2594.51)$ & $845.12(428.00-1730.77)$ & 0.002 \\
\hline ApoCII $\mu \mathrm{g} \cdot \mathrm{mL}^{-1}$ & $263.82(11.59-1794.55)$ & 165.01 (LLOD-757.13) & 0.007 \\
\hline ApoCIII $\mu \mathrm{g} \cdot \mathrm{mL}^{-1}$ & $305.80(157.92-646.63)$ & $203.18(112.44-398.44)$ & 0.005 \\
\hline ApoE $\mu \mathrm{g} \cdot \mathrm{mL}^{-1}$ & $973.58(454.16-1749.37)$ & $600.91(311.08-1058.87)$ & 0.001 \\
\hline
\end{tabular}

Data are presented as median (interquartile range), unless otherwise stated. CRP: C-reative protein; MIP: macrophage inflammatory protein; Apo: apolipoprotein; LLOD

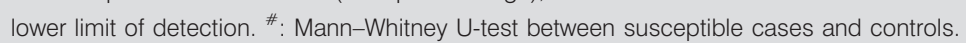

Utilising a nested case-cohort design, we were able to identify CVD serum biomarkers drawn within 6 months of September 11, 2001, that predicted if an exposed individual was likely to recover lung function or progress to lung disease. These biomarkers were expressed during disease evolution and so reflect processes leading to WTC-LI susceptibility or resistance. The observation that CVD biomarkers predict changes in lung function is consistent with a growing body of evidence that pulmonary vascular disease occurs early in COPD. This study emphasises the utility of serum stored in the aftermath of a disaster. This insight into protein expression may guide future mechanistic and therapeutic studies designed to blunt the impact of the worldwide COPD epidemic.

TABLE 5 Relative risk models predicting resistance or susceptibility to World Trade Center lung injury

\begin{tabular}{|c|c|c|c|c|c|}
\hline Analyte & Cut-off point ${ }^{\#}$ & \multicolumn{2}{|c|}{ Sample fraction $n / N$} & \multicolumn{2}{|c|}{ Relative risk $(95 \% \mathrm{Cl})$} \\
\hline \multicolumn{6}{|l|}{ Single analyte } \\
\hline $\mathrm{MPO} \mathrm{ng} \cdot \mathrm{mL}^{-1}$ & $\leqslant 105.26$ & $31 / 68$ & $31 / 124$ & $2.37(1.26-4.44)$ & $2.92(1.40-6.08)$ \\
\hline $\mathrm{sVCAM} n g \cdot \mathrm{mL}^{-1}$ & $\geqslant 1568.65$ & $30 / 68$ & $31 / 124$ & $2.51(1.34-4.71)$ & $2.26(1.10-4.64)$ \\
\hline $\mathrm{MPO} \mathrm{ng} \cdot \mathrm{mL}^{-1}$ & $\leqslant 105.26$ & $31 / 68$ & $31 / 124$ & $2.39(1.26-4.56)$ & $2.89(1.37-6.10)$ \\
\hline $\mathrm{sVCAM} n g \cdot \mathrm{mL}^{-1}$ & $\geqslant 1568.65$ & $30 / 68$ & $31 / 124$ & $2.53(1.34-4.82)$ & $2.24(1.07-4.70)$ \\
\hline AUC & & & & $0.647(0.564-0.731)$ & $0.830(0.774-0.887)$ \\
\hline \multicolumn{6}{|l|}{ Susceptible } \\
\hline \multicolumn{6}{|l|}{ Single analyte } \\
\hline $\mathrm{CRP} \mathrm{mg} \cdot \mathrm{dL}^{-1}$ & $\geqslant 2.45$ & $58 / 66$ & $85 / 124$ & $3.33(1.45-7.63)$ & $3.00(1.20-7.47)$ \\
\hline ApoCIII $\mu \mathrm{g} \cdot \mathrm{mL}^{-1}$ & $\geqslant 1058.87$ & $29 / 66$ & $31 / 124$ & $2.35(1.25-4.43)$ & $2.71(1.29-5.70)$ \\
\hline ApoE $\mu \mathrm{g} \cdot \mathrm{mL}^{-1}$ & $\geqslant 268.71$ & $33 / 66$ & $31 / 124$ & $3.00(1.60-5.64)$ & $3.03(1.46-6.30)$ \\
\hline \multicolumn{6}{|l|}{ Multi-analyte } \\
\hline ApoAll $\mu \mathrm{g} \cdot \mathrm{mL}^{-1}$ & $\geqslant 1772.87$ & $36 / 66$ & $30 / 124$ & $3.51(1.80-6.87)$ & $3.85(1.79-8.26)$ \\
\hline $\mathrm{CRP} \mathrm{mg} \cdot \mathrm{dL}^{-1}$ & $\geqslant 2.45$ & $58 / 66$ & $85 / 124$ & $4.01(1.65-9.79)$ & $3.93(1.43-10.79)$ \\
\hline MIP-4 $\mathrm{ng} \cdot \mathrm{mL}^{-1}$ & $\geqslant 194.90$ & $18 / 66$ & $59 / 124$ & $0.33(0.16-0.66)$ & $0.26(0.11-0.59)$ \\
\hline AUC & & & & $0.767(0.692-0.842)$ & $0.858(0.796-0.919)$ \\
\hline
\end{tabular}

MPO: myeloperoxidase; sVCAM: soluble vascular cell adhesion molecule; AUC: area under the curve; CRP: C-reative protein; MIP: macrophage inflammatory protein; Apo: apolipoprotein. \#: based on the 75th percentile of the controls for all analytes except MIP-4 (50th percentile), MPO (25th percentile) and CRP $\geqslant 2.45 \mathrm{mg} \cdot \mathrm{dL}-1$; ": risk without adjusting for any confounders; ${ }^{+}$: adjusted for age at September 11, 2001 (9/11), body mass index at subspecialty pulmonary examination, exposure group and pre-9/11 forced expiratory volume in $1 \mathrm{~s} \%$ predicted. 


\section{SUPPORT STATEMENT}

Funding was provided by: NIH: K23HL084191 (A. Nolan), K24A1080298 and RO1HL057879 (M.D. Weiden), T32 ES007267 (B. Naveed and S.J. Cho); National Center for Advancing Translational Sciences of the National Institutes of Health: UL1 TR000038, U01CA008617 and RO1HL090316 (W.N. Rom); and NIOSH/CDC: (U10-OH008243, U10-OH008242) and 1 UL1RR029893.

\section{STATEMENT OF INTEREST}

None declared.

\section{ACKNOWLEDGEMENTS}

We are thankful to the FDNY rescue workers for their selfless dedication

\section{REFERENCES}

1 Mills NL, Tornqvist $\mathrm{H}$, Gonzalez MC, et al. Ischemic and thrombotic effects of dilute diesel-exhaust inhalation in men with coronary heart disease. N Engl J Med 2007; 357: 1075-1082.

2 Tornqvist H, Mills NL, Gonzalez M, et al. Persistent endothelial dysfunction in humans after diesel exhaust inhalation. Am J Respir Crit Care Med 2007; 176: 395-400.

3 Naveed B, Weiden MD, Kwon S, et al. Metabolic syndrome biomarkers predict lung function impairment: a nested casecontrol study. Am J Respir Crit Care Med 2012; 185: 392-399.

4 Broekhuizen R, Wouters EF, Creutzberg EC, et al. Raised CRP levels mark metabolic and functional impairment in advanced COPD. Thorax 2006; 61: 17-22.

5 Gan WQ, Man SF, Senthilselvan A, et al. Association between chronic obstructive pulmonary disease and systemic inflammation: a systematic review and a meta-analysis. Thorax 2004; 59: 574-580.

6 Peters A, Dockery DW, Muller JE, et al. Increased particulate air pollution and the triggering of myocardial infarction. Circulation 2001; 103: 2810-2815.

7 Peters A, von Klot S, Heier M, et al. Exposure to traffic and the onset of myocardial infarction. N Engl J Med 2004; 351: 1721-1730.

8 Wellenius GA, Schwartz J, Mittleman MA. Air pollution and hospital admissions for ischemic and hemorrhagic stroke among medicare beneficiaries. Stroke 2005; 36: 2549-2553.

9 Wellenius GA, Yeh GY, Coull BA, et al. Effects of ambient air pollution on functional status in patients with chronic congestive heart failure: a repeated-measures study. Environ Health 2007; 6: 26

10 Wellenius GA, Coull BA, Batalha JR, et al. Effects of ambient particles and carbon monoxide on supraventricular arrhythmias in a rat model of myocardial infarction. Inhal Toxicol 2006; 18: 1077-1082.

11 Wellenius GA, Schwartz J, Mittleman MA. Particulate air pollution and hospital admissions for congestive heart failure in seven United States cities. Am J Cardiol 2006; 97: 404-408.

12 Dominici F, Peng RD, Bell ML, et al. Fine particulate air pollution and hospital admission for cardiovascular and respiratory diseases. JAMA 2006; 295: 1127-1134.

13 Cosio MG, Saetta M, Agusti A. Immunologic aspects of chronic obstructive pulmonary disease. New Engl J Med 2009; 360: 2445-2454.

14 Davies DE, Wicks J, Powell RM, et al. Airway remodeling in asthma: new insights. J Allergy Clin Immunol 2003; 111: 215-225.

15 Hoek G, Brunekreef B. Acute effects of a winter air pollution episode on pulmonary function and respiratory symptoms of children. Arch Environ Health 1993; 48: 328-335.

16 Brunekreef B, Hoek G. The relationship between low-level air pollution exposure and short-term changes in lung function in Dutch children. J Exposure Analysis Environ Epidemiol 1993; 3: Suppl. 1, 117-128.
17 Kumar S, Aldrich K. Overcoming barriers to electronic medical record (EMR) implementation in the US healthcare system: a comparative study. Health Informatics J 2010; 16: 306-318.

18 Koenig W. Inflammation and coronary heart disease: an overview. Cardiol Rev 2001; 9: 31-35.

19 Hoffmeister A, Rothenbacher D, Bazner U, et al. Role of novel markers of inflammation in patients with stable coronary heart disease. Am J Cardiol 2001; 87: 262-266.

20 Sin DD, Man SF. Why are patients with chronic obstructive pulmonary disease at increased risk of cardiovascular diseases? The potential role of systemic inflammation in chronic obstructive pulmonary disease. Circulation 2003; 107: 1514-1519.

21 Taraseviciene-Stewart L, Scerbavicius R, Choe KH, et al. An animal model of autoimmune emphysema. Am J Respir Crit Care Med 2005; 171: 734-742.

22 Voelkel N, Taraseviciene-Stewart L. Emphysema: an autoimmune vascular disease? Proc Am Thorac Soc 2005; 2: 23-25.

23 Zureik M, Benetos A, Neukirch C, et al. Reduced pulmonary function is associated with central arterial stiffness in men. Am J Respir Crit Care Med 2001; 164: 2181-2185.

24 Tockman MS, Pearson JD, Fleg JL, et al. Rapid decline in FEV1. A new risk factor for coronary heart disease mortality. Am J Respir Crit Care Med 1995; 151: 390-398.

25 Mannino DM, Thorn D, Swensen A, et al. Prevalence and outcomes of diabetes, hypertension and cardiovascular disease in COPD. Eur Respir J 2008; 32: 962-969.

26 Friedman SM, Maslow CB, Reibman J, et al. Case-control study of lung function in World Trade Center Health Registry area residents and workers. Am J Respir Crit Care Med 2011; 184: 582-589.

27 Banauch GI, Hall C, Weiden M, et al. Pulmonary function after exposure to the World Trade Center collapse in the New York City Fire Department. Am J Respir Crit Care Med 2006; 174: 312-319.

28 Prezant DJ, Weiden M, Banauch GI, et al. Cough and bronchial responsiveness in firefighters at the World Trade Center site. $N$ Engl J Med 2002; 347: 806-815.

29 Sikora AL, Wilson DJ, Aldrich CC, et al. Kinetic and inhibition studies of dihydroxybenzoate-AMP ligase from Escherichia coli. Biochemistry 2010; 49: 3648-3657.

30 Rodriguez-Roisin R, Drakulovic M, Rodriguez DA, et al. Ventilation-perfusion imbalance and chronic obstructive pulmonary disease staging severity. J Appl Physiol 2009; 106: 1902-1908.

31 Liebow AA. Pulmonary emphysema with special reference to vascular changes. Am Rev Respir Dis 1959; 80: 67-93.

32 Caplan-Shaw CE, Yee H, Rogers L, et al. Lung pathologic findings in a local residential and working community exposed to World Trade Center dust, gas, and fumes. J Occup Environ Med 2011; 53: 981-991.

33 King MS, Eisenberg R, Newman JH, et al. Constrictive bronchiolitis in soldiers returning from Iraq and Afghanistan. $N$ Engl J Med 2011; 365: 222-230.

34 Wanger J, Clausen JL, Coates A, et al. Standardisation of the measurement of lung volumes. Eur Respir J 2005; 26: 511-522.

35 Herbert R, Moline J, Skloot G, et al. The World Trade Center disaster and the health of workers: five-year assessment of a unique medical screening program. Environ Health Perspect 2006; 114: $1853-1858$

36 Miller MR, Hankinson J, Brusasco V, et al. Standardisation of spirometry. Eur Respir J 2005; 26: 319-338.

37 Weiden MD, Ferrier N, Nolan A, et al. Obstructive airways disease with air trapping among firefighters exposed to World Trade Center dust. Chest 2010; 137: 566-574.

38 Pepys MB, Hirschfield GM. C-reactive protein: a critical update J Clin Investig 2003; 111: 1805-1812.

39 Nolan A, Naveed B, Comfort AL, et al. Inflammatory biomarkers predict airflow obstruction after exposure to World Trade Center dust. Chest 2012; 142: 412-418. 
40 Danesh J, Wheeler JG, Hirschfield GM, et al. C-reactive protein and other circulating markers of inflammation in the prediction of coronary heart disease. N Engl J Med 2004; 350: 1387-1397.

41 Ridker PM, Hennekens $\mathrm{CH}$, Buring JE, et al. C-reactive protein and other markers of inflammation in the prediction of cardiovascular disease in women. N Engl J Med 2000; 342: 836-843.

42 Cirillo DJ, Agrawal Y, Cassano PA. Lipids and pulmonary function in the Third National Health and Nutrition Examination Survey. Am J Epidemiol 2002; 155: 842-848.

43 Pinto-Plata VM, Mullerova H, Toso JF, et al. C-reactive protein in patients with COPD, control smokers and non-smokers. Thorax 2006; 61: 23-28.

44 Yuditskaya S, Tumblin A, Hoehn GT, et al. Proteomic identification of altered apolipoprotein patterns in pulmonary hypertension and vasculopathy of sickle cell disease. Blood 2009; 113: $1122-1128$.
45 Vulcano M, Struyf S, Scapini P, et al. Unique regulation of CCL18 production by maturing dendritic cells. J Immunol 2003; 170: 3843-3849.

46 Azzaoui I, Yahia SA, Chang Y, et al. CCL18 differentiates dendritic cells in tolerogenic cells able to prime regulatory $\mathrm{T}$ cells in healthy subjects. Blood 2011; 118: 3549-3558.

47 Salvi S, Blomberg A, Rudell B, et al. Acute inflammatory responses in the airways and peripheral blood after short-term exposure to diesel exhaust in healthy human volunteers. Am J Respir Crit Care Med 1999; 159: 702-709.

48 Rundle AG, Vineis P, Ahsan H. Design options for molecular epidemiology research within cohort studies. Cancer Epidemiol Biomarkers Prev 2005; 14: 1899-1907.

49 Miettinen O. Design options in epidemiologic research. An update. Scand J Work Environ Health 1982; 8: Suppl. 1, 7-14.

50 Prentice RL. On the design of synthetic case-control studies. Biometrics 1986; 42: 301-310. 